\section{Contamination from satellites}

SIR-Radioastronomers must contend with many forms of man-made interference. Although some relief is provided by locating radio observatories in regions remote from industrial activity, our most important protection is the allocation by the International Telecommunication Union of frequency bands where transmissions which could disturb astronomical measurements are restricted. With the proliferation of artificial satellites and the approaching saturation of the electromagnetic spectrum, the preservation of these quiet bands assumes even greater importance.

In this context it is disturbing to realize that one particularly important band, the $1660.5-1670.0-\mathrm{MHz}$ band, which contains spectral lines of interstellar and circumstellar $\mathrm{OH}$, is already seriously contaminated with spurious emissions from satellites. Interference was first noticed in May 1989 while observing $\mathrm{OH}$ in supernova remnants', and a systematic investigation of its origin was begun. It was soon apparent from the temporal behaviour of the signals, from their polarization and from measurements of doppler shift, that the signals originated in satellites. A strong interfering signal at $1667.4900 \mathrm{MHz}$ was subsequently identified with satellites in the US global positioning system (GPS) ${ }^{2}$. Similar signals at $1660.9275,1661.4900,1663.2250$, $1663.2725,1664.9600$ and $1668.3350 \mathrm{MHz}$ have since been identified as originating in the Soviet Union's GLOSNASS navigational satellite system ${ }^{3}$.

Each navigation satellite broadcasts a 'spread spectrum' signal which produces strong sidebands well beyond the allocated frequencies. Dixon has explained the sideband structure ${ }^{4}$. Those narrow sidebands which fall within the radioastronomy bands produce spurious 'spectral lines' with flux densities over $10 \mathrm{kJy}$ $\left(10^{-22} \mathrm{~W} \mathrm{~m} \mathrm{~m}^{-2} \mathrm{~Hz}^{-1}\right)$. This is roughly a million times stronger than some of the astronomical spectral lines studied with our 26-m radio telescope. The spurious signals are so strong that they are regularly detected in sidelobes of the receiving antenna. In general, interference received in near sidelobes shows up in a single integration period (say $10 \mathrm{~min}$ ) and can be rejected. A more serious problem concerns spurious signals picked up in far sidelobes which are only apparent after many hours of integration and may then be indistinguishable from spectral features in the astronomical source being studied. I suspect this has already occurred in some of our preliminary studies.

For the GPS system, frequencies of the $n$th sidebands are $1575.42 \pm 10.23 n \mathrm{MHz}$. Only one of these frequencies lies in the $1660.5-1670.0-\mathrm{MHz}$ band, but the
GLONASS system produces many such frequencies. For GLONASS, the frequencies of the $n$th sidebands are given by $1602 .+0.5625 m \pm 5.11 n \mathrm{MHz}$, where $m$ takes a different value in the range 1 to 24 for each of 24 different satellites. When this system is complete it will produce 42 separate spurious signals in this radioastronomy band. During February 1990, I have confirmed that five of these satellites generate strong spurious signals in this band. The figure shows the spectrum of one of the GLONASS satellites, obtained by pointing the antenna directly at the satellite while scanning a single $10-\mathrm{kHz}$ wide channel across the band. As well as the three spikes, two of which are within the radioastronomy band, there are broad humps between the spikes and narrower shoulders close to the spikes. Power in these humps will contaminate continuum and extra-galactic spectral measurements while the spikes contaminate galactic spectral-line measurements.

I have examined ten of the presently active GPS satellites, six of which emit strong spurious emission in the ninth side-band at $1667.49 \mathrm{MHz}$ and four of which do not. The six offending satellites were all launched before 1986 . The fact that all GLONASS satellites and most of the GPS satellites emit considerable power in the 'protected' radioastronomy band indicates a lack of appreciation by system designers for the extremely low flux levels of astronomical radio sources

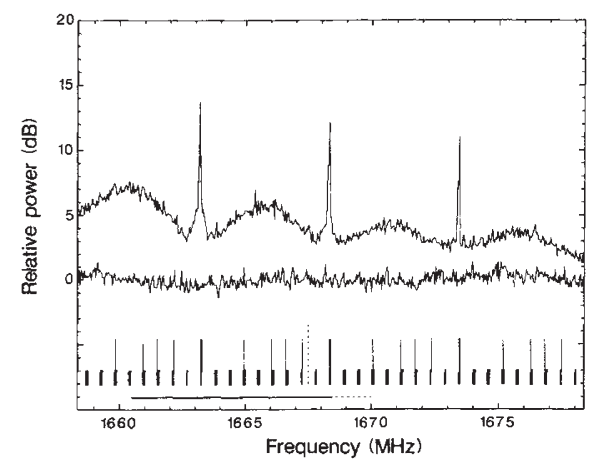

The upper trace is a spectrum of a GLONASS satellite in and near the radioastronomy band showing the 10th, 11th and 12th sidebands resulting from 'spread spectrum' modulation of the central frequency $1612.125 \mathrm{MHz}$. The lower trace was recorded with the antenna pointed about 10 degrees away from the satellite. Short vertical lines indicate frequencies where similar spikes will occur if the entire 24-satellite system is completed. Longer, vertical lines mark frequencies emitted by satellites which are now transmitting. Most GPS satellites emit a strong spurious signal at the frequency shown by the dashed vertical line. The band $1660.5-1668.4 \mathrm{MHz}$, where radioastronomy and passive space research share exclusive primary allocation is indicated by a solid horizontal line; the dashed extension indicates a shared band. and the high sensitivity which a radio telescope achieves through long integration times. On the other hand, the absence of this type of interference from recently launched US satellites indicates that suppressing such spurious emission is technically feasible.

The problem of pollution of radioastronomy bands is already serious ${ }^{5}$. In particular, establishment of the GLONASS system has now seriously curtailed radio studies of important classes of stars ${ }^{6}$. Both the GPS and the GLONASS systems were designed for the same purpose, the precise navigation of military craft, and neither is complete. Both, particularly the GLONASS system, are incompatible with continued observation of $\mathrm{OH}$ in astronomical sources. I strongly urge that future systems be compatible with the needs of radioastronomy and that existing systems causing interference be phased out.

\section{Dominion Radio Astrophysical}

JOHN GALT

\section{Observatory,}

Herzberg Institute of Astrophysics,

National Research Council,

Box 248 Penticton,

British Columbia V2A 6K3, Canada

1. Green, D.A. Mon. Not. R. astr. Soc. 238, 737-751 (1989)

2. Spilker, J.J. J J J inst. Navig. 25, 121-146 (1978).

3. Daly, P. J. Navig. 41, 186-198 (1988)

4. Dixon, R.C. Spread Spectrum Systems 2nd edn (Wiley, New York, 1984).

5. Cohen, R.J. Space Policy 5, 91-93 (1989)

6. Galt, J.A. in Proc. Colloq. Int. astr. Un. No. 122 (Washing ton, DC, in the press).

\section{Transmission of leukaemia}

SIR-There has been much recent discussion about the risk of leukaemia in children whose fathers had been occupationally exposed to radiation ${ }^{1.2}$. One crucial point, the possibility of an internal dose burden to the male gonads, has been neglected. The basic requirement for such an event is that relevant radionuclides are retained selectively by testicular tissue.

This phenomenon was investigated experimentally more than 20 years ago by Lüning et $a l^{3}{ }^{3}$, who observed mutagenic effects in the offspring of male mice which had incorporated strontium-90. These authors postulated that strontium could be present in nucleoproteins of the germ cells as a metal ion. A similar possibility was also raised recently by Riley and Willson ${ }^{+}$, who speculated that zinc, for instance, may be retained in the gonads and/or may be a vehicle for transporting short-lived radionuclides to critical local sites such as testicular tissue. A further hypothesis - with special reference to chemical agents - has been discussed in News and Views by Evanss.

We have demonstrated that yttrium-90, the daughter product of strontium- 90 , is 